\title{
Candida endophthalmiltis following penetrating keratoplasty in patient with negative donor rim; a case report with review of literature for diagnosis and treatment of fungal endophthalmitis
}

\section{Abstract}

This is a case of young patient presented with granulomatous anterior and posterior uveitis, which turned to be fungal endophthalmitis after penetrating keratoplasty. Her symptoms were undetected because she was on systemic and topical steroids. The patient is a 25 years old Caucasian female, previously medically free of any disease, who was admitted to the Eye Clinic at the Jordan University Hospital, Amman, Jordan, for left penetrating keratoplasty and severe keratoconus. After an initial improvement in her vision and a smooth postoperative course, she presented with drop of vision, photophobia, and non-specific eye pain. On examination she was found to have anterior granulomatous uveitis. She was started on systemic steroids and the topical steroids were increased in intensity. The initial systemic workup for granulomatous anterior uveitis was negative. However, culture of the aqueous was positive for Candida galibrata, but the donor rim was negative. Later the patient developed vitritis despite being on systemic fluconazole and topical amphotericin B. She was treated with intravitreal amphotericin B. The vitritis improved, but vitreous opacities developed which deteriorated her vision. A parsplana vitetrectony was done. Her final visual acuity remained poor because of opacified graft. The patient's unfortunate case represents a Candida endophthalmitis after penetrating keratoplasty despite being medically a healthy person.

\section{Introduction}

Corneal transplantation is considered a proved way of treatment of various corneal disorders [1]. Fungal keratitis and endoophthalmitis
Mouna Al Saad',

Sohaib Alkowni ${ }^{1}$

1 Department of Special Surgery, School of Medicine, The University of Jordan, Amman, Jordan

Contact information:

Mouna Al-Saad.

”" eye_maas@yahoo.com

Keywords

Candida Endophthalmiltis; Penetrating Keratoplasty; Negative Donor Rim. 
is a rare but well known complication that occurs post transplantation [2]. It had an incidence of 1.4 per 10000 transplants. Culture can detect presence of fungal keratitis and endoophthalmiltis [2]. Fungal infections of post keratoplasty can lead to severe complications [1].

Candida chorioretinitis typically presents as several, small, creamy white, circumscribed chorioretinal lesions with overlying vitreous inflammation. In certain cases, these chorioretinal lesions may be surrounded by hemorrhage, giving them the appearance of a white-centered hemorrhage. Within the area of the lesion, the retinal vessels may be sheathed, and the vitreous opacities resemble fluffy balls, and they may be linked to each other by strands giving them the string of pearls appearance. In case the infection is not detected the disease advances, epiretinal membranes may develop, leading to vitreoretinal traction and retinal detachment. The lesions often heal with treatment, but chorioretinal scarring may be developed in the areas of prior inflammation. Two thirds of patients have bilateral disease, and more than one half of patients have vitreous involvement. Iridocyclitis often is present, and fungal infection of the iris and ciliary body is rare [3].

\section{Case report}

A 25 year old female patient diagnosed with keratoconus in both eyes, with mild degree in the right eye and severe one in her left eye. The right eye was doing well with rigid contact lens obtaining a visual acuity of 0.7. However, her left eye had a visual acuity of CF 1m, which did not improve with contact lens or glasses. The keratometric reading was 70 and 77 dioptrers in the left eye for which a penetrating keratoplasty was indicated.

She was medically healthy person and was not on any medications. An uneventful penetrating keratoplasty was done in her left eye. Her immediate postoperative visual acuity was 0.2. She was started on topical prednesilone acetate every two hours and topical ofloxcacin every four hours. She had a smooth postoperative course, so her topical steroids were tapered and a visual acuity of 0.4 was gained. Few months later the patient developeda drop in her vision and severe anterior uveitis, which was thought that it is a rebound uveitis due to tapering of her topical steroids. As a result, the topical steroid was increased in frequency to every one hour, and after a fair response, the patient was started on oral steroids predisilone acetate of $1 \mathrm{mg} / \mathrm{kg}$.

The patient showed a good response initially and her visual acuity improved, but she went back with a drop in her visual acuity. After examination her visual acuity, she was found to have granulomatous anterior uveitis with mutton fat keratic precipitate. Her B scan was negative for vitritis. A workup was done including chest $X$-ray, Purified Protein Derivative (PPD) skin test for tuberculosis, $C B C$ with differential, Angiotensin Converting Enzyme level, Cytoplasmic Antinuetrophil Cytoplasmic Autoantibody, While waiting for the results, which turned to be negative, the oral steroids were maintained. Later, the patient developed a white fluffy matter in the anterior chamber, with more severe inflammation. An aqueous tap was done for gram stain, fungal and bacterial culture and susceptibility tests. Culture of the donner bottom was done which turned to be negative. There was budding yeast in the gram stain and the culture turned to be positive for Candida glabrata. The patient was referred to the infectious disease unit and was started on oral fluconazole and topical amphotrecin B $(50 \mathrm{mg} / \mathrm{ml})$. During follow up, a breached anterior capsule was noted with opacified lens. A history of trauma was present, which was denied by the patient latter.

A lens aspiration with intraocular lens implantation and culture of the aqueous was obtained again. It turned to be positive for Candida glabrata. The patient was maintained on oral fluconazole and topical amphotrecin B. The patient visual acuity improved to 0.2. Four months later, the patient developed mild discomfort and drop in her visual 
acuity to counting fingers $(C F)$ at $1 \mathrm{~m}$. A posterior uveitis developed vitreous tap for fungal culture and susceptibility and intravitreal amphotrecin B of $0.1 \mathrm{ml}$ $(50 \mathrm{mg} / \mathrm{ml})$ was given. The vitreous culture turned to be negative. The patient improved initially and the vitritis subsided but vitreous opacities developed and a thick retro-lental membrane developed and the IOL lens was displaced anteriorly, so the visual acuity was decreased to hand motion (HM). A parsplana vitrectomy was done and a second vitreous culture was done and intravitreal amphotericin $B$ was given. During the surgery, there were thick vitreous opacities with very adherent posterior hyaloid but the retina looked healthy. The fungal culture was negative again. Although her smooth postoperative recovery, her visual acuity remained HM due to opacified graft.

\section{Discussion}

Although fungal keratitis and endophthalmitis post keratoplasty is very rare, it has a very poor prognosis $[1,3]$. Patients who develop Candida endophthalmitis have risk factors like intravenous drug abuse being the most common one, other risks include; long standing indwelling catheters, postpartum women, premature infants, patients undergoing hyper alimentation, history of recent abdominal surgery, and patients with debilitating diseases like diabetes mellitus, post organ transplantation or malignancies $[1,3]$.

The symptoms of Candida endophthalmitis may include loss of vision which may be unrecognized in cases of endogenous endophthalmitis, visual acuity may not be affected if the lesion is peripheral, red eye, and photophobia, pain, floaters or scotoma may be present. Candida endophthalmitis typically presents as several small well demarcated peripheral creamy lesions surrounded by vitreous inflammation, Roth spots, which are areas of chorioretinitis surrounded by hemorrhages, may be present. The nearby vessel may be sheathed. The vitreous may be involved and vitritis typically present as illdefined balls of inflammation connected together by strands resembling strings of pearls. In unrecognized cases epiretinal membrane develops resulting in vitreous traction and epiretinal membrane formation. Healed chorioretinal lesions leave scared areas which may be complicated by choroidal endovascular membrane. The infection is bilateral in two third of patients and vitritis is present in half of the patients. Iridocyclitis is usually present and hypopyon may develop, although presence of infection of the iris or the ciliary body is rare.

Most cases of fungal endophthalmitis are due to Candida albicans $[1,3]$. Candida glabrata is also a common ocular pathogen with increasing frequency and predilection for eyes with corneal transplant [4]. Most reported cases were also associated with a positive culture of the donned cornea. In half of the cases there is a latent period of several months before the fungal infection is recognized and due to delay in diagnosis, most cases required re-grafting [4-8]. The most commonly isolated yeast in infectious keratitis is C. albicans [8]. Also, C. glabrata related infections are documented and such cases are difficult to treat because C. glabrata is resistant to most antifungal agents used in treatment of $C$. albicans [4]. Differential diagnoses include postoperative endophthalmitis, acute retinal necrosis, acute complications of sarcoidosis, toxoplasmosis, leukemia, interstitial keratitis and tuberculosis. The diagnosis of fungal endophthalmitis should be suspected in any immunocompromised patient present with vitritis and chorioretenitis.

The diagnosis of fungal infections in the ophthalmic practice requires the presence of pathology. A presumptive diagnosis is possible when the fungus is isolated by culture from any part of the body; like blood, cerebrospinal fluid, urine, sputum associated with presence of classical intraocular findings. In suspected cases of endogenous endophthalmitis, cultures with direct examination for fungi using Giemsa, Gomori-Methenamine-Silver (GMS) and 
Periodic-Acid Schiff stains should be requested. In the present study, the presence of the fungal infection was documented by culture [8]. C. glabrata was isolated on two different occasions using aqueous sample for culture. Fungal culture on Sabourauds dextrose agar can be positive in $44 \%$ to $70 \%$ of clinically diagnosed cases. Vitectomy samples are more yielding than vitreous needle samples[9], any growth should be considered as significant rather than contamination and the samples should be kept for 4-6 weeks to insure that slow growing fungi are not missed [8].

A modern technique of diagnosis of fungal endophthalmitis is using polymerase chain reaction (PCR). PCR is a rapid and high sensitive method and helps in early differentiation between bacterial and fungal endophalmitis. PCR was used successfully to identify Candida species from intra ocular fluids [10]. In addition, DNA microarray is a modern rapid helpful method of diagnosis [11]. Candida species grows well on Sabourauds dextrose media at room temperature and 37C without cycloheximide as white and pasty colonies after incubation 2-4 days. A simple wet preparation can reveal the presence of yeast cells. Germ-tube test can be used to differentiate between C. alblicans and other Candida spp. ChromCandida agar is frequently used to confirm the presence of all clinically common Candida species [12].

Various drugs are used in the treatment of fungal endophthalmitis. Drugs include, amphotrecin B, fluconazole, ketokenazole, miconazole, flucytosine, itraconazole and caspofungin. But the best initial treatment for endogenous fungal endophthalmitis has not been established. However, both amphotericin B and fluconazole are recommended [13]. Systemic amphotericin has been the drug of choice because of its broad spectrum activity, but the penetration of the vitreous cavity is limited, and doses of 5 to $10 \mathrm{mg}$ intravitreal amphotericin is commonly used. Retinal toxicity was reported in animal models eith such doses [13]. Fluconazole and flucytosine have good intraocular penetration, but Candida species have already high resistance to flocytosine. A recent drug is voriconazole which is administered systematically, has a good intra vitreal penetration and is not retino toxic when given intra vitreal in a concentration up to $25 \mathrm{mg} / \mathrm{ml}$ [14]. Antifungal susceptibility test in vitro using minimum inhibitory concentrations (MICs) does not always correlate with in vivo values, for that reason the MIC of fungal drugs in vitro should only be used as a guideline [15].

The echinocandines (caspofungin, micafungin and anidulafungin) are new drugs that work by inhibiting D-glucan synthase, which is an essential enzyme involved in fungal wall synthesis. They have activity against most species of Candida and Aspergillus. Capsofungin and amphotrecin B are equally effective in candedimia and fungal endophthalmitis, but fewer side effects were reported with capsofungin compared to amphotrecin B [16]. Gauthier et al. [14], reported a case of Candida endophthalmitis that failed to respond to capsofungin due to poor penetration into the vitreous. The role of capsofungin in the treatment on Candida endophthalmitis remains limited. Al Assiri et al. [17], recommended routine culture of the donor corneal rim at the time of surgery to detect potential source of late onset fungal infections like C. glabrata. Vislisel et al. [18], concluded that positive donor rim cultures are uncommon but carry an unacceptably high risk of post graft fungal infection. This process can be reduced with prophylactic antifungal treatment in positive cultures [19]. We have used amphotericin B topically and intraviteally in our patient and showed a good response. Grueb et al. [13], found that topical and intracameral application of amphotericin B is sufficient and safe in the therapy of C. glabrata endophthalmitis post keratoplasty in 26 old man. 


\section{References}

1. Koji kitazawa, Koichi Wakimasu, Kazuhito Yonedo, Berrie Lliakis, Chie Sotozono and Shigeru Kinoshita. A case of fungal keratitis and endoopthalmitis post penetrating keratoplasty resulting from fungal contamination of the donor cornea. American J Ophthalmol Case Reports 2017; (5)103-106.

2. Eye banking statistical report, Eye bank association of America,2015, Washington, DC 20036.

3. Keyhani K, Seedor JA, Shan MK, Terranciano AJ, and Ritterband DC. The incidence of fungal keratitis and endophthalmitis following penetrating keratoplasty. Cornea 2005; 24(3):288-91.

4. Caldwell, John R. Perfect, Alan N. Carlson and Alan D Proia. Candida Galibrata endophthalmitis following keratoplasty. J Cataract Refract Surg 2009; 35(3):598-602.

5. Sutphin JE, Pfaller MA, Hollis RJ and Wagoner MD. Donor to host transmission of Candida Albicans after corneal transplantation. Am J Ophthalmol 2002; 134:120-121.

6. Kitzmann AS,Wagoner MD, Sayed NA \& Goins KM. Donor related candida keratitis after Descemet Stripping automated endothelial keratoplasty. Cornea 2009; 28:228-230.

7. Tappeeiner C, Goldblum D, Zimmerli S, Fux C, and Frueh B.E. Donor to host transmission of Candida Galibrata to both recipients of corneal transplants of the same donor. Cornea 2009; 28(2)228-230.

8. Yamazoe K, Den S, Yamaguchi T, Tanaka Y and Shimazaki J. Severe donor related Candida keratitis after Descemet stripping automated endothelial keratoplasty. Graefes Arch Clin Exp Ophthalmol 2011; 249(10):1579-82.

9. William A, Spitzer MS, Dueter C, Blumenstock , Partsch M, VoykovB, et al. Outcome of Primary Transconjunctival 23G Vitrectomy in the Diagnosis and Treatment of Presumed Endogenous Fungal Endophthalmitis. Ocul Immunol Inflamm 2012; 6:321-6.

10. Anand A,Madhavan $H$, Neelam $V$, and Lily $T$, Use of polymerase chain reaction in the diagnosis of fungal endophthalmitis. Ophthalmology 2001; 108(2):326-30.

11. Sakai T, Kohzaki K, watanabe A, TsuneokkaH, Shimadadzu M, Use of DNA microarray analysis in diagnosis of bacterial and fungal endophthalmitis. Clin Ophthalmol 2012; 6:321-6.

12. Shehabi AA, Nazal SA, Dajani N. Putative virulence factors of Candida species colonizing respiratory tracts of patients. Microb Ecology Health Dis 2004; 16:214-217.

13. Grueb M,Rohrbach JM, and Zierhut M. Amphotericin B in the therapy of C. Galibrata after penetrating keratoplasty. Cornea 2006; 25(10):1243-4

14. Gauthier GM, Nork TM Prince R, Andes D. subtherapuitic ocular penetration of Capsufungin and associated treatment failure in Candida Albicans Endophthalmitis. Clinc Infect Dis 2005; 41:(3) e27-8.
15. Khan FA, Slain D, KaKhoo RA. Candida Endophthalmitis: focus on current and future antifungal treatment options. Pharmacotherapy 2007; 27(12):1711-21.

16. Mora-Duarte J, Betts R., Rotstein C, Colombo Al, ThompsonMoya L, Smietana J. Comparison of Capsofungin and Amphotrecin B for intensive candidiasis. N Engl J Med 2002; 347(25):2020-9.

17. Al Assiri A, Al-Jastaneah S, Al-Khalaf A, Al-Fraikh $H$, and Wagoner MD. Late onset donor to host transmission of Candida Galibrata following coneal transplantation. Cornea 2006; 25(1):123-5.

18. Vislisel JM,Goins K, Wagonmer MD, Schmidt, GA, Aldrich Skeie $B T$, Reed CR, Zimmeman MB, and Greiner MA. Incidence and outcomes of positive Donor corneoscleral rim fungal Cultures after Keratoplasty. Ophthalmology 2017; 124(1)36-42.

19. Hind M. Alkaatan, AzzaMaktabi, Mosa Al Harbyand Ali A. Al-Rajhi. Candida Parapsilosis corneal graft infection from asingle eye center: histopathologic report of two cases. Saudi J Ophthalom 2015; 29: 303-306.

\section{Publish in The International}

Arabic Journal of Antimicrobial Agents

The Journal is an open access peer-reviewed journal that publishes scientific papers about all aspects of antimicrobials. The journal will publish original research articles, reviews, brief reports and case reports dealing with basic and clinical antibacterial agents, antiviral, antiprotozoals, antituberculuous, antifungal and antihelminthes agents. All manuscripts must be prepared in English, and are subject to a rigorous and fair peer-review process. Accepted papers will immediately appear online. The journal aims to advance the knowledge, attitude and the research of chemotherapy in the Arabic world in cooperation with international, national scientific and public societies as well as research centers with similar aims and objectives. 